\title{
Burden of psychiatric disorder in young adulthood and life outcomes at age 30
}

\author{
Sheree J. Gibb, David M. Fergusson and L. John Horwood
}

\section{Background}

Psychiatric disorders are common during young adulthood and comorbidity is frequent. Individual psychiatric disorders have been shown to be associated with negative economic and educational outcomes, but few studies have addressed the relationship between the total extent of psychiatric disorder and life outcomes.

\section{Aims}

To examine whether the extent of common psychiatric disorder between ages 18 and 25 is associated with negative economic and educational outcomes at age 30, before and after controlling for confounding factors.

\section{Method}

Participants were 987 individuals from the Christchurch Health and Development Study, a longitudinal study of a birth cohort of individuals born in Christchurch, New Zealand, in 1977 and followed to age 30. Linear and logistic regression models were used to examine the associations between psychiatric disorder from age 18 to 25 and workforce participation, income and living standards, and educational achievement at age 30, before and after adjustment for confounding factors.

\section{Results}

There were significant associations between the extent of psychiatric disorder reported between ages 18 and 25 and all of the outcome measures (all $P<0.05$ ). After adjustment for confounding factors, the associations between psychiatric disorder and workforce participation, income and living standards remained significant (all $P<0.05$ ), but the associations between psychiatric disorder and educational achievement were not significant (all $P>0.10$ ).

\section{Conclusions}

After due allowance had been made for a range of confounding factors, psychiatric disorder between ages 18 and 25 was associated with reduced workforce participation, lower income and lower economic living standards at age 30

\section{Declaration of interest}

None.
Psychiatric illness is a common experience during the early adult years. The recent National Comorbidity Survey Replication estimated that the 12-month prevalence of DSM-IV psychiatric disorder in the USA among young adults (ages 18-29) was $43.8 \%{ }^{1}$ The disorders with the highest 12 -month prevalence among this age group included anxiety disorders (22.3\%), substance use disorders $(22.0 \%)$ and mood disorders (12.9\%). The high rates of psychiatric disorder among young adults are of concern given that psychiatric illness has been shown to be associated with a range of negative life outcomes, including lower educational achievement, decreased employment, lower incomes and lower standard of living. ${ }^{2-4}$ These negative outcomes have been associated with a range of different psychiatric disorders, including depression, ${ }^{5,6}$ anxiety disorders ${ }^{7-9}$ and substance use disorders. ${ }^{10,11}$ However, there has been some debate over whether psychiatric illness causes negative life outcomes, or whether the associations can be explained by confounding factors that influence both the likelihood of developing psychiatric disorder and the risk of negative life outcomes. Some studies have reported that the associations between psychiatric illness and life outcomes can be explained by confounding factors, ${ }^{12}$ but others have reported that significant associations remain after adjustment for confounding factors. ${ }^{2,4,5}$ For example, Fergusson et $a l^{5}$ reported that depression between the ages of 16 and 21 was significantly associated with a range of negative life outcomes at age 25 , including mental health outcomes, welfare dependence and unemployment. These associations remained significant even after adjustment for a range of background factors, including family background, history of physical and sexual abuse, neuroticism, gender and comorbid psychiatric disorders.

Although previous studies have examined the associations between specific psychiatric illnesses and life outcomes while controlling for confounding factors, few have examined whether additional episodes of psychiatric disorder have increasingly negative effects on life outcomes. This is surprising given that comorbidity in psychiatric illness is common. For example, the National Comorbidity Survey Replication found that, of those individuals who reported having a psychiatric illness within the past 12 months, $45.0 \%$ reported having two or more illnesses and $22.9 \%$ reported three or more illnesses. ${ }^{1}$ The aims of the present study were to examine the associations between the extent of psychiatric disorder during young adulthood and a range of life outcomes at age 30, including workforce participation, living standards, income and educational achievement. In contrast to most of the previous literature in this area, this study considered the impact of the extent of common psychiatric illness on life outcomes at age 30, rather than examining the effects of a specific psychiatric disorder. The data are drawn from the Christchurch Health and Development Study, a longitudinal study of a birth cohort of individuals born in Christchurch, New Zealand, in 1977 and followed to age 30. The use of a rich longitudinal data source allows the associations between psychiatric illness and life outcomes to be adjusted for a wide range of confounding factors, including family background, academic ability, life events and individual characteristics.

\section{Method}

\section{Participants}

Data were collected as part of the Christchurch Health and Development Study, a consecutive series of 1265 individuals born in Christchurch, New Zealand, in 1977 and followed to age 30. Participants were followed up at birth, 4 months, 1 year, yearly 
until age 16, then at ages 18,21, 25 and 30. Data were collected using a combination of semi-structured interviews, standardised tests and teacher reports. The methodology and major findings of this study have been reviewed previously. ${ }^{13,14}$ All information was collected on the basis of signed consent from study participants.

Over the course of the study there has been a gradual loss of participants as a result of participant refusal, death and loss to follow-up. At age 30, 987 participants remained in the study, representing $80.0 \%$ of the surviving cohort at age 30 .

The present analysis is based on a sample of 950 participants who were assessed at age 30 and had complete data available on the extent of psychiatric disorder between the ages of 18 and 25 . This sample represented $77.7 \%$ of the surviving cohort at age 30 .

\section{Psychiatric disorder}

At ages 21 and 25, participants were questioned about the extent to which they had suffered from symptoms of depression, mania, generalised anxiety disorder, panic disorder, social phobia, specific phobias, agoraphobia, substance use, misuse and dependence, and antisocial personality disorder in the period since the preceding interview. Questions were drawn from the Composite International Diagnostic Interview (version 3, revised) ${ }^{15}$ and were modified slightly in order to assess psychiatric symptoms over the entire period since the previous interview. Responses to these questions were used to determine the presence or absence of a series of psychiatric disorders at age 30 based on the instructions in the CIDI (version 3, revised) manual and DSM-IV ${ }^{16}$ criteria (see Fergusson et al for a more detailed explanation of the criteria used). ${ }^{17}$

To measure the extent of psychiatric disorder during young adulthood, six dichotomous measures were constructed representing the presence or absence of each of three disorder types during each of two time periods (age 18-21 and age 2125). These disorder types were: major depression; any anxiety disorder (generalised anxiety disorder, panic disorder, agoraphobia, social phobia or specific phobia); and any substance dependence disorder (alcohol dependence, cannabis dependence or other substance dependence, excluding nicotine dependence).

These dichotomous measures were summed to create a scale ranging from 0 to 6 , with 0 representing those with no psychiatric diagnoses during the period from age 18 to 25 , and 6 representing those meeting criteria for all disorders during both time periods. For simplicity, this scale was condensed to a four-level scale representing the number of psychiatric disorders over the time period: none; one; two to three; or four or more. The correlation between the condensed and original scales was strong $(d=0.98)$.

Consideration was given to creating an extended scale measure of psychiatric disorder. This scale was the sum of 18 dichotomous variables representing the presence of each of the nine disorders that were used to compose the disorder categories for the original scale (major depression, generalised anxiety disorder, panic disorder, social phobia, agoraphobia, specific phobia, alcohol dependence, cannabis dependence and other substance dependence) in two time periods (age 18-21, and age 21-25). These variables were summed to create a scale ranging from 0 to 18 , with 0 representing those with no psychiatric diagnoses during the period from age 18 to 25 , and 18 representing those meeting criteria for all disorders during both time periods. Scores on this scale were strongly correlated with those on the previous six-level scale $(d=0.94)$ and also the condensed four-level scale $(d=0.90)$. Due to the strong correlations between the three versions of the scale the rest of this paper uses the condensed four-level version of the scale for simplicity.
Consideration was also given to including the presence or absence of conduct disorder/antisocial personality disorder during the periods from 18 to 21 and from 21 to 25 . However, analysis revealed that these diagnoses were not significantly related to the outcome variables once due allowance was made for confounding factors. Therefore, they were not included.

\section{Outcome measures}

Information obtained from the interview at age 30 was used to construct the following outcome measures.

\section{Paid employment}

Participants working in paid employment for any number of hours per week at age 30 were classified as being in paid employment. Overall, $83.2 \%$ of participants were in paid employment.

\section{Full-time employment}

Participants working in paid employment for 30 or more hours per week were classified as being in full-time employment. Overall, $74.2 \%$ of participants were in full-time employment.

\section{Hours worked}

The total number of hours worked in paid employment per week at age 30 was calculated. Where weekly hours varied, participants were asked to estimate an average. The mean was $34.0 \mathrm{~h}$ (s.d. $=19.1$ ).

\section{Welfare dependence}

Participants who reported receiving a sickness, invalid, domestic purposes or unemployment government benefit at age 30 were classified as being welfare dependent. Overall, $8.4 \%$ of participants were welfare dependent.

\section{Total personal income}

Participants were asked to report their total net weekly personal income, from wages, benefits, investments and all other sources, after tax and deductions, at age 30. Incomes in currencies other than New Zealand dollars were converted to New Zealand dollars using Purchasing Power Parity rates for 2007. ${ }^{18}$ The mean was $\$ 767.0$ (s.d. $=\$ 738.1$ ).

\section{Owning a home}

Participants who reported that they owned the home in which they were living at age 30 (regardless of whether or not they had a mortgage on the home) were classified as owning a home. Overall, $36.8 \%$ of participants owned a home.

\section{Economic living standards}

As part of the interview at age 30, participants completed the Economic Living Standards Index Short Form, ${ }^{19}$ which measures the material aspects of well-being that are reflected in a person's consumption and personal possessions. Higher scores on this index indicate higher economic living standards. The mean score was 24.9 (s.d. = 5.2). 


\section{University degree}

At age 30, participants were asked if they had ever attained a degree from a New Zealand or overseas university. Overall, $29.0 \%$ of participants had attained a university degree.

\section{Tertiary qualifications}

At age 30, participants were asked if they had ever attained a postschool (tertiary) qualification that took at least 3 months of study to attain. Overall, $69.0 \%$ of participants had one.

\section{Overall highest educational achievement}

The highest level of educational achievement reached by age 30 was classified on a seven-point scale where 1 was no qualifications, 2-5 represented increasing levels of high school qualifications, 6 was attending university and 7 was a university degree. The mean score was 4.2 (s.d. $=2.2$ ).

\section{Covariate factors}

A series of covariate factors were included in the regression models. These covariate factors were: family socioeconomic status at birth; maternal age; maternal educational achievement; exposure to childhood adversity age $0-10$; family living standards age $0-10$; parental changes to age 15 ; childhood physical punishment; childhood sexual abuse; total Wechsler Intelligence Scale for Children -Revised (WISC-R) ${ }^{20}$ IQ score; age of leaving secondary school; Test of Scholastic Abilities (TOSCA) ${ }^{21}$ score; teacher-rated academic performance age 11-13; gender; motherrated conduct problems age 16; neuroticism age 14; and deviant peer affiliations age 15. Detailed descriptions of these covariate factors can be found in the online supplement.

\section{Statistical methods}

Associations between the extent of psychiatric disorder during young adulthood and life outcomes at age 30 were examined using linear regression models (for continuous outcomes) or logistic regression models (for dichotomous outcomes). Regression modelling was conducted in two stages. First, outcomes were modelled as a function of the extent of psychiatric disorder between the ages of 18 and 25. Second, regression models from the first stage were extended to include a series of covariate factors. All covariate factors were initially included, and the model was subsequently refined by removing those factors that were not significant $(P<0.05)$ predictors of the outcome measure. The refined model was used to estimate adjusted mean or proportion scores. For continuous outcomes, adjusted mean scores were calculated using the least squares method. For dichotomous outcomes, adjusted proportions were calculated using the methods described by Lee. ${ }^{22}$

Associations between individual categories of psychiatric disorder and life outcomes at age 30 were examined using regression models in the same way as described earlier, except that the variable representing the number of psychiatric disorders was replaced with a variable representing the number of episodes of the relevant disorder (depression, any anxiety disorder or any substance dependence disorder excluding nicotine dependence) between the ages of 18 and 25 . These variables were constructed by summing the dichotomous measures of the presence or absence of the relevant disorder in the periods from 18 to 21 and from 21 to 25 .

\section{Sample size and sample bias}

The gradual loss of participants over time raises questions about the extent to which the results of the present study may be affected by sample bias due to sample loss. To examine the effects of sample loss, missing data were imputed and the results re-analysed using the 'PROC MI' and 'PROC MIANALYZE' procedures in SAS 9.1 for Windows. These procedures use methods of multiple imputation, considered to be one of the best techniques for dealing with missing data (see Rubin, ${ }^{23,24}$ Schafer, ${ }^{25}$ Schafer \& Graham), ${ }^{26}$ to impute missing outcomes and then use these outcomes in the analysis to adjust standard error estimates under the assumption that there was no bias in the estimation of the outcomes.

The results of the analysis using the imputed data were not substantively different from those using the original data and, in all cases, led to the same conclusions. This suggests that the results of the present study were not significantly affected by sample bias due to sample loss. Therefore, the remainder of this paper presents only the results obtained using the original (reduced) sample.

\section{Results}

Table 1 shows the associations between the extent of psychiatric disorder reported between the ages of 18 and 25, and a series of workforce participation, economic and educational outcomes at age 30 .

\begin{tabular}{|c|c|c|c|c|c|c|}
\hline \multirow[b]{2}{*}{ Outcomes at age 30} & \multicolumn{6}{|c|}{ Number of psychiatric disorders, aged $18-25$} \\
\hline & $\begin{array}{c}0 \\
(n=488)\end{array}$ & $\begin{array}{c}1 \\
(n=223)\end{array}$ & $\begin{array}{c}2-3 \\
(n=214)\end{array}$ & $\begin{array}{c}4+ \\
(n=53)\end{array}$ & $B$ (s.e.) & $P$ \\
\hline \multicolumn{7}{|l|}{ Workforce participation } \\
\hline Working in paid employment, \% & 88.6 & 83.0 & 76.9 & 64.2 & $-0.45(0.09)$ & $<0.001$ \\
\hline Working in full-time employment, \% & 80.3 & 74.2 & 65.4 & 52.8 & $-0.41(0.08)$ & $<0.001$ \\
\hline Hours worked per week, mean (s.d.) & $36.5(17.4)$ & $34.0(19.1)$ & $30.4(20.5)$ & $23.8(20.2)$ & $-3.50(0.63)$ & $<0.001$ \\
\hline \multicolumn{7}{|l|}{ Income and living standards } \\
\hline Welfare dependent, \% & 3.8 & 10.1 & 12.5 & 22.6 & $0.62(0.12)$ & $<0.001$ \\
\hline Total net weekly personal income, \$NZ: mean (s.d.) & $847.1(922.5)$ & $746.2(563.7)$ & $676.1(531.7)$ & $574.5(402.3)$ & $-88.29(25.38)$ & $<0.001$ \\
\hline Owning a home, \% & 43.4 & 34.1 & 28.4 & 34.0 & $-0.26(0.07)$ & $<0.001$ \\
\hline Economic living standards index, mean (s.d.) & $26.2(4.2)$ & $24.4(5.2)$ & $23.2(5.7)$ & $22.2(6.1)$ & $-1.43(0.17)$ & $<0.001$ \\
\hline \multicolumn{7}{|l|}{ Educational achievement } \\
\hline Attained university degree, \% & 33.4 & 30.9 & 25.2 & 11.3 & $-0.27(0.08)$ & $<0.001$ \\
\hline Attained tertiary qualification, $\%$ & 73.1 & 66.4 & 65.4 & 66.0 & $-0.16(0.07)$ & 0.04 \\
\hline Overall highest educational achievement, mean (s.d.) & $4.6(2.2)$ & $4.4(2.3)$ & $4.1(2.3)$ & $3.7(2.2)$ & $-0.25(0.08)$ & 0.002 \\
\hline
\end{tabular}


Psychiatric disorder during young adulthood was common, with $50.1 \%$ of participants experiencing at least one psychiatric disorder (depression, anxiety disorder or substance dependence) between the ages of 18 and 25. Many participants experienced more than one episode of psychiatric disorder during young adulthood. Of those who experienced any psychiatric disorder during young adulthood, $54.5 \%$ experienced two or more episodes of disorder. It should be noted that these multiple episodes of disorder may indicate either multiple episodes of a single disorder or episodes of different disorders.

To examine the associations between the extent of psychiatric disorder during young adulthood and life outcomes at age 30, data were fitted with linear regression models (for continuous outcomes) or logistic regression models (for dichotomous outcomes) in which the outcome measure was modelled as a function of the number of psychiatric disorders during the period from age 18 to age 25 (see Method for details). This analysis revealed that increasing episodes of psychiatric disorder during young adulthood were significantly associated with a range of negative life outcomes. These negative outcomes included decreased likelihood of being in paid employment $(P<0.0001)$, decreased likelihood of being in full-time employment $(P<0.0001)$, fewer hours worked per week $(P<0.0001)$, higher likelihood of being welfare dependent $(P<0.0001)$, lower total personal income $(P<0.0006)$, lower likelihood of owning a home $(P<0.0005)$, lower economic living standards $(P<0.0001)$, lower likelihood of attaining a university degree $(P<0.0007)$, lower likelihood of attaining a tertiary qualification $(P<0.04)$ and lower overall highest educational achievement $(P<0.002)$.

The likelihood of experiencing negative life outcomes increased as the extent of psychiatric disorder during young adulthood increased. For all of the outcome measures, those with no episodes of psychiatric disorder had the least negative outcomes, while those with four or more episodes of psychiatric disorder had the most negative outcomes.

Table 1 shows that psychiatric disorders during early adulthood were associated with reduced workforce participation, lower income and living standards, and lower educational achievement outcomes at age 30, with increasing episodes of disorder being associated with increasingly negative outcomes. However, the associations between psychiatric disorder in young adulthood and life outcomes at age 30 may be confounded by variables that are associated with both psychiatric disorder during young adulthood and life outcomes at age 30. To examine this, associations between the number of psychiatric disorders from age 18 to age 25 and the covariate factors were tested for statistical significance using a chi-squared test (see Methods and the online supplement for a full list of covariate factors). A more detailed summary of the results of this analysis can be found in online Table DS1. An increasing number of psychiatric disorders was significantly associated with: younger maternal age $(P<0.003)$; greater exposure to childhood adversity $(P<0.0001)$; lower family living standards during childhood $(P<0.0002)$; greater numbers of parental changes $(P<0.0001)$; increased likelihood of childhood sexual abuse $(P<0.0001)$, increased exposure to physical punishment during childhood $(P<0.0001)$; leaving school at younger ages $(P<0.0001)$; female gender $(P<0.0004)$; higher levels of conduct problems during adolescence $(P<0.0001)$; higher neuroticism scores $(P<0.0001)$; and greater likelihood of associating with deviant peers during adolescence $(P<0.0001)$.

The preceding analyses showed that psychiatric disorder during young adulthood was significantly associated with a range of family background, academic ability and individual characteristics. This suggests that the associations between psychiatric disorder during young adulthood and life outcomes at age 30 need to be adjusted for covariate factors. To achieve this, the regression models were extended to include a series of covariate factors related to family background, life events and individual characteristics (see Method and the online supplement for details).

Table 2 shows the adjusted associations between psychiatric disorder in young adulthood and life outcomes at age 30 . The associations have been adjusted for covariate factors. The table also shows the covariate factors that were statistically significant $(P<0.05)$ predictors in the regression models.

For all of the outcome measures, the adjusted regression coefficients in Table 2 are smaller than the regression coefficients in Table 1, indicating that adjusting for covariate factors reduced the associations between psychiatric disorder and life outcomes. However, most of the associations between the extent of psychiatric disorder during young adulthood and life outcomes at age 30 remained statistically significant $(P<0.05)$ after adjustment for covariate factors. After adjustment, increasing numbers of episodes of psychiatric disorder between the ages of 18 and 25 were associated with: decreasing likelihood of paid

Table 2 Covariate-adjusted associations ${ }^{a}$ between psychiatric disorder during young adulthood and life outcomes at age 30

\begin{tabular}{|c|c|c|c|c|c|c|c|}
\hline & \multicolumn{4}{|c|}{ Number of psychiatric disorders age 18-25 } & \multirow[b]{2}{*}{ B (s.e.) } & \multirow[b]{2}{*}{$P$} & \multirow[b]{2}{*}{$\begin{array}{l}\text { Significant } \\
\text { covariates }^{\text {b }}\end{array}$} \\
\hline & $\begin{array}{c}0 \\
(n=488)\end{array}$ & $\begin{array}{c}1 \\
(n=223)\end{array}$ & $\begin{array}{c}2-3 \\
(n=214)\end{array}$ & $\begin{array}{c}4+ \\
(n=53)\end{array}$ & & & \\
\hline \multicolumn{8}{|l|}{ Workforce participation } \\
\hline Working in paid employment, \% & 87.8 & 83.9 & 79.2 & 73.5 & $-0.34(0.11)$ & 0.003 & $1,3,4,5,6,15$ \\
\hline Working in full-time employment, \% & 78.8 & 74.4 & 69.5 & 64.2 & $-0.29(0.10)$ & 0.005 & $1,2,3,4,6,12$ \\
\hline Hours worked per week, mean & 35.4 & 33.5 & 31.6 & 29.7 & $-1.89(0.70)$ & 0.008 & $1,3,4,6$ \\
\hline \multicolumn{8}{|l|}{ Income and living standards } \\
\hline Welfare dependent, \% & 4.7 & 7.8 & 12.5 & 19.4 & $0.56(0.15)$ & $<0.001$ & 4,6 \\
\hline Total net weekly personal income, \$NZ: mean & 819.5 & 764.0 & 708.5 & 653.0 & $-55.48(25.64)$ & 0.04 & $1,2,10$ \\
\hline Owning a home, \% & 41.5 & 36.2 & 31.2 & 26.6 & $-0.23(0.08)$ & 0.005 & $1,9,10,16$ \\
\hline Economic living standards index, mean & 25.8 & 24.8 & 23.8 & 22.8 & $-1.02(0.19)$ & $<0.001$ & $4,6,10,11$ \\
\hline \multicolumn{8}{|l|}{ Educational achievement } \\
\hline Attained university degree, \% & 29.8 & 27.4 & 25.0 & 22.7 & $-0.21(0.13)$ & 0.10 & $2,4,7,8,10,12,13$ \\
\hline Attained tertiary qualification, $\%$ & 71.2 & 68.8 & 66.3 & 63.8 & $-0.13(0.09)$ & 0.16 & $1,4,9,10,12$ \\
\hline Overall highest educational achievement, mean & 4.3 & 4.3 & 4.2 & 4.2 & $-0.03(0.07)$ & 0.66 & $1,6,7,8,9,10,12,13$ \\
\hline $\begin{array}{l}\text { a. Adjusted means and proportions have been estimated fron } \\
\text { b. Significant covariates }(P<0.05) \text { : } 1 \text {, gender; } 2 \text {, teacher-rated } \\
7 \text {, age left school; } 8 \text {, deviant peer affiliations, age } 16 ; 9 \text {, mater } \\
13 \text {, family socioeconomic status at birth; } 14 \text {, parental changes }\end{array}$ & $\begin{array}{l}\text { ne regressi } \\
\text { ademic pe }\end{array}$ & $\begin{array}{l}\text { dels using } \\
\text { nce, age } 1\end{array}$ & $\begin{array}{l}3 \text {, childho } \\
\text { mily living } \\
\text { nduct prob }\end{array}$ & $\begin{array}{l}\text { od for m } \\
\text { ical abuse } \\
\text { ds, age } 0 \\
\text { age } 16 .\end{array}$ & $\begin{array}{l}\text { s) or the methoc } \\
\text { exposure to ch } \\
11 \text {, childhood s }\end{array}$ & $\begin{array}{l}\text { described } \\
\text { hood adv } \\
\text { tual abuse }\end{array}$ & $\begin{array}{l}\text { y Lee } 22 \text { (for proportions). } \\
\text { sity; } 5 \text {, neuroticism; } 6, \text { IQ; } \\
\text { 2, Test of Scholastic Ability; }\end{array}$ \\
\hline
\end{tabular}


employment $(P<0.003)$; decreasing likelihood of full-time employment $(P<0.005)$; fewer hours worked per week $(P<0.008)$; increasing rates of welfare dependence $(P<0.0001)$; decreased total personal income $(P<0.04)$; lower likelihood of owning a home $(P<0.005)$; and lower economic living standards $(P<0.0001)$. However, after adjustment for covariate factors there were no longer significant associations between psychiatric disorders during young adulthood and any of the educational achievement outcomes (attaining a university degree, attaining a tertiary qualification, and overall highest educational achievement, all $P>0.10$ ). Inspection of the covariates revealed that the models for the educational achievement outcomes tended to include a greater number of covariate factors than the other models. They also tended to include more covariate factors related to family socioeconomic background. This suggests that the relationships between psychiatric disorder and educational achievement can be explained by differences in covariate factors including family socioeconomic background that are associated with both educational achievement and the likelihood of developing psychiatric disorder.

The analyses presented thus far have examined the associations between the total extent of psychiatric disorder during young adulthood and life outcomes at age 30. However, they have not considered whether different psychiatric disorders may have different impacts on life outcomes. To examine this, data were fitted with multiple linear regression models (for the continuous outcomes) or multiple logistic regression models (for the dichotomous outcomes) in which the outcome measure was modelled as a function of a single psychiatric disorder type (depression, anxiety disorder or substance use disorder) and the series of covariate factors. The full results of these regression models can be found in online Table DS2. Overall, the three psychiatric disorder types had similar influences on life outcomes at age 30. Depression and substance dependence were significantly $(P<0.05)$ associated with almost all of the workforce participation and income and living standards outcomes, with the only exceptions being the associations with total personal income, which were marginally significant $(P<0.09)$. Anxiety disorders were also associated with most of the workforce participation and income and living standards outcomes, with the only exceptions being paid employment, owning a home, and hours worked per week, where the associations were not significant $(P>0.11)$. In almost all cases, the psychiatric disorders were not significantly associated with the educational achievement outcomes $(P>0.22)$, with the only exception being the association between substance dependence and degree attainment $(P<0.03)$.

To further examine whether different disorder categories had different effects on life outcomes, the regression models that were fitted to the data in Table 2 were extended in two ways.

First, in order to examine whether including information about specific disorder types made any difference to the conclusions of the main analysis, the original models (from Table 2) were extended to include three dichotomous variables representing the presence of a diagnosis of each disorder type (depression, anxiety disorder and substance dependence disorder) over the period from age 18 to age 25 . In almost all cases, these variables were not significant predictors in the model $(P<0.05)$, and in the few cases where they were significant predictors, their inclusion in the regression models made no difference to the conclusions of the original analysis reported in Table 2 .

Second, in order to examine whether different combinations of disorder types had different effects on life outcomes, the original models (from Table 2) were extended to include all possible two-way interactions between the three disorder types. In no case were any of these interactions statistically significant
$(P>0.05)$, suggesting that different combinations of disorder type had similar effects on life outcomes.

\section{Discussion}

This study examined the associations between the extent of common psychiatric disorder during young adulthood and life outcomes at age 30 using data from the Christchurch Health and Development Study, a longitudinal study of a birth cohort of individuals born in 1977 and followed for 30 years. The major findings from the present study are discussed below.

Psychiatric disorder during young adulthood (age 18-25) was common, with approximately half of the study participants meeting DSM-IV criteria for depression, anxiety disorder or substance dependence during this time period. Among those meeting criteria for psychiatric disorder, multiple episodes of disorder were frequent, with $65.3 \%$ of participants having two or more episodes of disorder.

Increasing numbers of psychiatric disorders during young adulthood were significantly associated with a range of negative life outcomes at age 30, including reduced workforce participation, lower income and living standards and lower educational achievement. However, psychiatric disorder during young adulthood was also associated with a range of confounding factors including family background, academic ability and childhood abuse. After adjustment for these confounding factors, psychiatric disorder during young adulthood was significantly associated with reduced workforce participation, lower income and lower living standards, but was not significantly associated with educational achievement. The relationships between psychiatric disorder and educational achievement could be explained by covariate factors, and particularly, factors associated with family socioeconomic background, which influenced both educational achievement and the likelihood of developing psychiatric disorders.

Whereas previous studies have examined the relationships between specific psychiatric disorders and life outcomes, there has been little research examining the cumulative effect of multiple episodes of psychiatric disorder on life outcomes. The results from this study show that increasing episodes of psychiatric disorder have increasingly negative effects on life outcomes, even after adjustment for confounding factors. In many cases these effects are substantial. For example, after adjustment for confounding factors, the rate of welfare dependence was more than four times higher among those with four or more episodes of psychiatric disorder compared with those with no psychiatric disorder. Similarly, those with four or more episodes of psychiatric disorder worked almost 6 fewer hours per week than those with no psychiatric disorder, and earned \$166 less per week.

\section{Implications}

The findings from this study revealed that psychiatric disorder during young adulthood is common, and is predictive of a range of negative life outcomes including reduced workforce participation, lower income, and lower standard of living. This suggests a need for further improvements in the diagnosis and treatment of psychiatric illness in order to reduce the negative outcomes experienced by those with psychiatric disorder. However, the results of this study also revealed that those individuals most at risk of negative outcomes are not those who experience any specific psychiatric disorder, but rather, those who experience multiple episodes of disorder. This finding suggests that that there is a need to develop targeted interventions for those with multiple psychiatric disorders in 
order to reduce the risks of negative life outcomes among this group.

\section{Strengths and limitations}

A major strength of this study is that it used a rich longitudinal data-set that allowed the relationships between psychiatric disorder and life outcomes to be adjusted for a wide range of confounding factors. However, this study also had several limitations. Data were drawn from a birth cohort of individuals born at a specific time in a specific place, and therefore it is not clear to what extent the results from this cohort may generalise to other populations. Furthermore, the measure of extent of psychiatric disorder used in this study was limited and considered only the presence of depression, anxiety disorder or substance dependence. It did not include other disorders such as personality disorders, bipolar disorder or attention-deficit hyperactivity disorder. However, the relatively low rates of these disorders in the adult population ${ }^{1,27}$ means that it is unlikely that their inclusion would have a substantial impact on the results. Furthermore, given that significant associations between psychiatric disorder and life outcomes were found using an approximate measure of psychiatric disorder, it is likely that a more precise measure would increase the precision of the models and therefore strengthen, rather than weaken, the conclusions of the study. Finally, while the associations between the extent of psychiatric disorder and life outcomes were adjusted for a wide range of covariate factors, it remains possible that there are additional confounding variables that were not included in the models that could explain the associations.

Within these limitations, the results of this study suggest that those who experience psychiatric disorder during young adulthood are at increased risk of a range of negative life outcomes at age 30. The risk of negative outcomes increases as the total burden of psychiatric disorder increases, with those experiencing the most episodes of psychiatric disorder being at greatest risk of negative outcomes.

Sheree J. Gibb, PhD, David M. Fergusson, PhD, L. John Horwood, MSC Christchurch Health and Development Study, University of Otago, Christchurch School of Medicine and Health Sciences, Christchurch, New Zealand

Correspondence: Sheree J. Gibb, Christchurch Health and Development Study, University of Otago, Christchurch, PO Box 4345, Christchurch, New Zealand. Email: sheree.gibb@otago.ac.nz

First received 13 Dec 2009, final revision 30 Mar 2010, accepted 12 Apr 2010

\section{Funding}

This research was funded by grants from the Health Research Council of New Zealand, the National Child Health Research Foundation the Canterbury Medical Research Foundation and the New Zealand Lottery Grants Board. S.J.G. was supported by a University of Otago Division of Health Sciences Career Development Postdoctoral Fellowship and a University of Otago Postgraduate Publishing Bursary.

\section{References}

1 Harvard University School of Medicine. NCS-R Twelve-month Prevalence estimates. Harvard School of Medicine, 2005 (http:// www.hcp.med.harvard.edu/ncs/).
2 Ettner SL, Frank RG, Kessler RC. The impact of psychiatric disorders on labor market outcomes. Ind Labor Relat Rev 1997; 51: 64-81.

3 Kessler RC, Heeringa S, Lakoma MD, Petukhova M, Rupp AE, Schoenbaum M, et al. Individual and societal effects of mental disorders on earnings in the United States: results from the National Comorbidity Survey Replication. Am J Psychiatry 2008; 165: 703-11.

4 Kessler RC, Foster CL, Saunders WB, Stang PE. Social consequences of psychiatric disorders. I: Educational attainment. Am J Psychiatry 1995; 152 1026-32.

5 Fergusson DM, Boden JM, Horwood LJ. Recurrence of major depression in adolescence and early adulthood, and later mental health, educational and economic outcomes. Br J Psychiatry 2007; 191: 335-42.

6 Andrews B, Wilding JM. The relation of depression and anxiety to life-stress and achievement in students. Br J Psychol 2004; 95: 509-21.

7 Van Ameringen $M$, Mancini $C$, Farvolden $\mathrm{P}$. The impact of anxiety disorders on educational achievement. Anxiety Disord 2003; 17: 561-71.

8 Woodward $\amalg$, Fergusson DM. Life course outcomes of young people with anxiety disorders in adolescence. J Am Acad Child Adolesc Psychiatry 2001; 40: 1086-93.

9 Patel A, Knapp M, Henderson J, Baldwin D. The economic consequences of social phobia. J Affect Disord 2002; 68: 221-33.

10 Buchmueller TC, Zuvekas SH. Drug use, drug abuse, and labor market outcomes. Health Econ 1998; 7: 229-45.

11 Bray JW, Zarkin GA, Dennis ML, French MT. Symptoms of dependence, multiple substance use, and labor market outcomes. Am J Drug Alcohol Abuse 2000; 26: 77-99.

12 Fergusson DM, Woodward LJ. Mental health, educational and social role outcomes of adolescents with depression. Arch Gen Psychiatry 2002; 59: 225-31.

13 Fergusson DM, Horwood $\mathrm{L}$. The Christchurch Health and Development Study: review of findings on child and adolescent mental health. Aust $N \mathrm{ZJ}$ Psychiatry 2001; 35: 287-96.

14 Fergusson DM, Horwood $\amalg$, Shannon FT, Lawton JM. The Christchurch Child Development Study: a review of epidemiological findings. Paediatr Perinat Epidemiol 1989; 3: 278-301.

15 World Health Organization. Composite International Diagnostic Interview (CIDI). WHO, 1993.

16 American Psychiatric Association. Diagnostic and Statistical Manual of Mental Disorders (4th edn) (DSM-IV). APA, 1994.

17 Fergusson DM, Horwood L, Lynskey MT. Childhood sexual abuse and psychiatric disorders in young adulthood. Part II: Psychiatric outcomes of sexual abuse. J Am Acad Child Adolesc Psychiatry 1996; 35: 1365-74.

18 Organisation for Economic Co-operation and Development. Purchasing Power Parities (PPPS) for OECD Countries since 1980. OECD, 2007 (http://www.oecd.org/std/ppp).

19 Jensen J, Spittal M, Krishnan V. ELSI Short Form: User Manual For a Direct Measure of Living Standards. Ministry of Social Development, 2005.

20 Wechsler D. Wechsler Intelligence Scale for Children - Revised. Psychological Corporation, 1974.

21 Reid NA, Jackson PF, Gilmore A, Croft C. Test of Scholastic Abilities. Ministry of Education, 1981.

22 Lee J. Covariance adjustment of rates based on the multiple logistic regression model. J Chronic Dis 1981; 34: 415-26.

23 Rubin DB. Multiple Imputation for Nonresponse in Surveys. John Wiley \& Sons, 1987.

24 Rubin DB. Multiple imputation after 18+ years. J Am Stat Assoc. 1996; 91 473-89.

25 Schafer JL. Multiple imputation: a primer. Stat Methods Med Res 1999; 8: 3-15.

26 Schafer JL, Graham JW. Missing data: our view of the state of the art Psychol Methods 2002; 7: 147-77.

27 Kessler RC, Chiu WT, Demler O, Merikangas KR, Walters EE. Prevalence, severity, and comorbidity of 12-Month DSM-IV disorders in the National Comorbidity Survey Replication. Arch Gen Psychiatry 2005; 62: 617-27. 